\title{
Experimental study of externally prestressed segmental beam under torsion
}

\begin{abstract}
Externally prestressed segmental (EPS) concrete beams are widely used in the construction of bridge structures today. The EPS concept has become an attractive tool for rehabilitation and strengthening of existing bridges which have insufficient strength and/or excessive deflection and cracking. EPS bridges are affected by combined stresses (bending, shear, normal, and torsion) at the joint interface between the segments. This paper presents an experimental investigation of the structural behaviour of EPS bridge beams under combined stresses, i.e. bending, shear and torsional stresses. An experimental study of two different external tendon layouts, two types of joint between segments and different levels of torsional force applied at different load eccentricities was conducted. It was concluded that torsion reduced the vertical load and vertical deflection at the onset point of nonlinearity as well as at a failure load. Furthermore, the opening between segments was the reason behind the nonlinear behaviour of EPS beams. Torsion did not only alter the value of the failure load, tendon strain and deflection of the beams but it also altered the failure mechanism. Furthermore, due to the shear key, there was a difference between the onset points of nonlinearity load for the different tendon layouts and joint types.
\end{abstract}

Keyword: Externally prestressed; Segmental concrete bridge; Combined stresses; Torsion 